\title{
Synthesis and structure of temperature-sensitive nanocapsules
}

\author{
Monia Brugnoni ${ }^{1} \cdot$ Fabian Fink $^{1} \cdot$ Andrea Scotti $^{1} \cdot$ Walter Richtering $^{1}$ (D)
}

Received: 20 April 2020 / Revised: 2 June 2020 / Accepted: 2 June 2020 / Published online: 23 June 2020

(C) The Author(s) 2020

\begin{abstract}
The transport and systematic release of functional agents at specific areas are key challenges in various application fields. These make the development of micro- and nanocapsules, which allow for uptake, storage, and triggered release, of high interest. Hollow thermoresponsive microgels, cross-linked polymer networks with a solvent-filled cavity in their center, are promising candidates as triggerable nanocapsules, as they can adapt their size and shape to the environment. Their shell permeability can be controlled by temperature, while the cavity can serve as a storage place for guest species. Here, we present the synthesis and structural characterization of temperature-responsive microgels, which are deswollen at room temperature and swell upon moderate cooling, to facilitate potential encapsulation experiments. We present microgels made from poly( $N$-isopropylacrylamide-co-diacetone acrylamide), p(NIPAM-co-DAAM), possessing a volume phase transition temperature below room temperature. Their colloidal stability in the deswollen state can be enhanced by adding a swollen polymer shell made of poly( $N$-isopropylacrylamide), pNIPAM, as periphery. The synthesis of hollow double-shell microgels comprising a cavity surrounded by an inner p(NIPAM-co-DAAM) shell and an outer pNIPAM shell is established. The inner network enables the control of the shell permeability: the network is deswollen at room temperature and swells upon moderate cooling. The outer network guarantees for steric stability at room temperature. Light scattering techniques are employed for the characterization of the microgels. Form factor analysis reveals that the cavity of the nanocapsules persists at all swelling states, making it an ideal site for the storage of guest species.
\end{abstract}

Keywords Microgels $\cdot$ Hollow structures $\cdot$ Volume phase transition $\cdot$ Thermosensitivity $\cdot$ Light scattering

\section{Introduction}

"Smart" switchable microgels represent a promising type of materials as they reveal important features for potential applications as drug delivery systems [1,2]. Their response to external stimuli, e.g., temperature or $\mathrm{pH}$, enables to store guest species within the networks and protects the guests from the environment with the possibility of triggered release on demand [3-6].

Microgels are soft polymeric networks, which exhibit various responsive characteristics depending on their chemical composition. The most prominent and easily

Electronic supplementary material The online version of this article (https://doi.org/10.1007/s00396-020-04686-5) contains supplementary material, which is available to authorized users.

Walter Richtering

richtering@rwth-aachen.de

1 Institute of Physical Chemistry, RWTH Aachen University, 52056 Aachen, Germany controllable trigger for microgels is temperature [7, 8]. The best-studied temperature-sensitive microgels are based on poly $(N$-isopropylacrylamide) (pNIPAM) [9]. Typically, pNIPAM microgels are obtained from the polymerization of $N$-isopropylacrylamide (NIPAM) with a cross-linking agent to ensure the formation of a chemically cross-linked polymer network. The network density and, therefore, the stiffness and permeability can be tuned by controlling the amount of cross-linker during the polymerization [10]. The addition of co-monomers during the polymerization reaction allows to create microgels with distinct responsiveness, e.g., an additional $\mathrm{pH}$ sensitivity [11-13]. Furthermore, sophisticated microgel architectures can be produced by complex synthesis procedures to generate, e.g., core-shell [14-17], core-double-shell [1820], or hollow [20-22] structures.

Micro- and nanocapsules are under strong investigation aiming for various applications [23-25]. Hollow pNIPAM nanocapsules swollen in water can be produced by various techniques, e.g., in emulsions [26, 27], in microfluidic devices [28], or by removal of a sacrificial core [21, 22]. 
They possess high potential because the solvent-filled cavity provides a screened environment for guest species [3, 2932]. In addition, these capsules can potentially be used for a temperature-triggered uptake, storage, and release of macromolecules or small colloids. The cavity of the microgels then serves as a storage place for the guests, while the switchable permeability of the polymeric shell can be exploited for temperature-triggered encapsulation.

With a volume phase transition temperature (VPTT) of about $32{ }^{\circ} \mathrm{C}$ in water [9], pure pNIPAM microgels are rather difficult to employ for uptake and release experiments. The encapsulation would occur only well above room temperature making the handling of the deswollen state challenging. Therefore, we aim to synthesize hollow microgels with a VPTT slightly below room temperature such that the microgels are deswollen at room temperature and swell upon moderate cooling, e.g., in a refrigerator. This shift of the VPTT can be achieved by the addition of an appropriate co-monomer with distinct hydrophilicity when compared to NIPAM.

Diacetone acrylamide (DAAM) [33-36] and $N$-tertbutylacrylamide (NtBAM) [37-41] are examples of co-monomers that decrease the lower critical solution temperature (LCST) of polymers when being co-polymerized with acrylamides. This temperature drop results from an increase in the hydrophobic hydration of the polymer. Thus, the more co-monomer is in the network, the lower is the VPTT of the microgels until the thermoresponsive character known from pNIPAM is fully suppressed.

NtBAM is known to decrease the VPTT of the microgels when being co-polymerized with NIPAM [37, 40]. However, we reveal that the synthesis of hollow colloidally stable microgels is precluded and aggregation as well as a loss of network integrity result. As an alternative, we introduce p(NIPAM-co-DAAM) microgels. First, regular microgels are synthesized to determine the appropriate monomer composition for the desired manageable thermosensitivity: deswollen microgels at ambient conditions that swell upon moderate cooling. The poor colloidal stability is attempted to be improved in two distinct ways: (i) by increasing the electrostatic interaction through adding a surfactant during the precipitation polymerization and (ii) by increasing the steric interactions at room temperature by adding a swollen polymer shell as periphery. We identify a considerably improved stability of the microgels when adding steric interactions in terms of an outer pNIPAM shell. This provides the basis for the synthesis of the hollow microgels via core dissolution.

An intact cavity is crucial for storage applications. Its size can be tuned by the choice of the sacrificial core [22, 42, $43]$, the composition of the polymer shell [19, 20], and the environmental constraints as adsorbed to interfaces [44-46] or entrapped in highly crowded solutions [42, 47].
Here, silica nanoparticles are chosen as cores to perform a seeded precipitation polymerization to synthesize core-double-shell microgels as depicted in Fig. 1. The corresponding hollow microgels are obtained by core dissolution. They possess an inner p(NIPAM-co-DAAM) network, which is deswollen at room temperature and swells upon moderate cooling, and an outer pNIPAM periphery for steric stabilization at room temperature.

We demonstrate by means of light scattering that the comonomer DAAM is suitable to lower the VPTT and to form colloidally stable hollow microgels, which maintain a cavity at all swelling states.

\section{Experimental section}

\section{Materials}

Ethanol and sodium hydroxide were purchased from VWR. NIPAM was obtained from Acros Organics. NtBAM, DAAM, $N, N^{\prime}$-methylenebisacrylamide (BIS) and 3-Methacryloxypropyltrimethoxysilane (MPS) were obtained from Sigma-Aldrich. Potassium persulfate (KPS), sodium dodecyl sulfate (SDS), tetraethyl orthosilicate (TEOS), and ammonia solution (28-30\%) were purchased from Merck.

\section{Synthesis}

Regular p(NIPAM-co-DAAM) microgels were prepared from precipitation polymerization of various molar ratios of NIPAM:DAAM:BIS, namely 0.30:0.65:0.05 (referred to as 65\%DAAM) and $0.65: 0.30: 0.05$ (referred to as $30 \%$ DAAM). The monomer solutions were prepared according to Table 1 , preheated to $70^{\circ} \mathrm{C}$ under constant stirring and purged with nitrogen. The reactions were initiated by rapid addition of $2.1 \mathrm{mg}$ of KPS in $0.5 \mathrm{~mL}$ of water and let to proceed for $2 \mathrm{~h}$.

The synthesis of the 30\%DAAM microgels was repeated in the presence of $1.4 \mathrm{mg}$ of SDS in the monomer solution (30\%DAAM-Electrostatic) and by addition of a

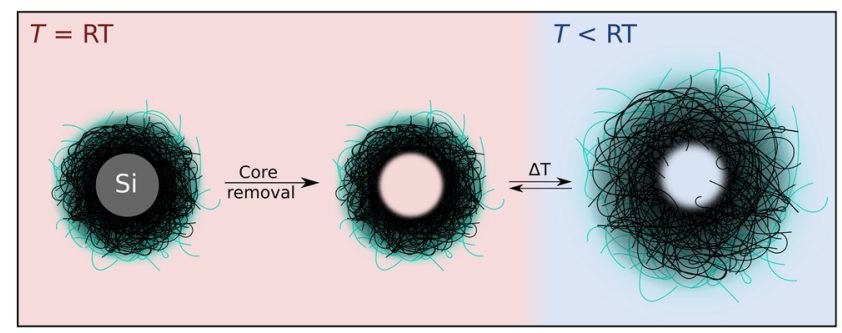

Fig. 1 Schematic representation of silica-core double-shell microgels with a deswollen inner shell (black) and a swollen outer shell (cyan) and the corresponding hollow microgels at room temperature (RT). The inner shell swells upon moderate cooling below room temperature 
Table 1 Composition of reaction solutions during synthesis of regular p(NIPAM-co-DAAM) and silica-core p(NIPAM-co-DAAM)shell pNIPAM-shell microgels

\begin{tabular}{llllc}
\hline Microgel & $\begin{array}{l}m_{\text {NIPAM }} \\
(\mathrm{mg})\end{array}$ & $\begin{array}{l}m_{\text {DAAM }} \\
(\mathrm{mg})\end{array}$ & $\begin{array}{l}m_{\text {BIS }} \\
(\mathrm{mg})\end{array}$ & $\begin{array}{l}V \\
(\mathrm{~mL})\end{array}$ \\
\hline $65 \%$ DAAM & 17.0 & 55.0 & 3.9 & 5 \\
30\%DAAM & 36.8 & 25.4 & 3.9 & 5 \\
CSS & 36.8 & 25.4 & 3.9 & 10 \\
\hline
\end{tabular}

pure pNIPAM periphery to the microgels by adding $5.9 \mathrm{mg}$ of NIPAM and $0.2 \mathrm{mg}$ of KPS in $0.5 \mathrm{~mL}$ of water $10 \mathrm{~min}$ after the start of the polymerization (30\%DAAM-Steric).

Silica nanoparticles with a radius of $60 \mathrm{~nm}$ serving as sacrificial cores have been prepared according to the Stöber synthesis [48]. $700 \mathrm{~mL}$ of ethanol were preheated to $60^{\circ} \mathrm{C}$. $63 \mathrm{~mL}$ of ammonia solution were added and let to equilibrate. The synthesis was initiated by adding $30 \mathrm{~mL}$ of TEOS to the reaction solution. After $24 \mathrm{~h}$, the nanoparticle suspension was cooled to room temperature. The nanoparticles were surface functionalized with MPS [22] and purified by threefold centrifugation at $5000 \mathrm{rpm}$ and redispersion in fresh ethanol.

Silica-core p(NIPAM-co-DAAM)-shell microgels with an outmost pNIPAM-shell (CSS) were prepared according to $30 \%$ DAAM with the pNIPAM periphery in presence of $0.5 \mathrm{~mL}$ of a silica nanoparticles suspension $(1.5 \mathrm{~g}$ in $10 \mathrm{~mL}$ of ethanol) and $2.9 \mathrm{mg}$ of SDS in the monomer solution (see Table 1). The reaction was initiated by the addition of a solution of $4.3 \mathrm{mg}$ of KPS in $0.5 \mathrm{~mL}$ of water. The core-double-shell microgels were purified by dialysis.

The corresponding hollow microgels were obtained by $\mathrm{NaOH}$ treatment of the core-double-shell microgels and subsequent dialysis in a refrigerator [22].

\section{Dynamic light scattering}

Dynamic light scattering was used to determine the hydrodynamic radii of the microgels as a function of temperature. An ALV setup connected to a goniometer and a HeNe laser with $\lambda=633 \mathrm{~nm}$ was used for all measurements. A programmable thermostat controlled the temperature of the toluene bath surrounding the sample. A minimum of 10 scattering angles were probed at each temperature with an acquisition time of $60 \mathrm{~s}$. The average diffusion coefficient, $D_{0}$, is obtained from the linear regression of the decay rate, $\Gamma$, from the second-order cumulant fit as a function of the magnitude of the scattering vector [49]. Finally, the Stokes-Einstein equation allows us to derive the hydrodynamic radius. Highly diluted samples were probed to prevent multiple scattering and interparticle interactions. The heating to cooling cycle does not show any hysteresis, i.e., the swelling-deswelling of microgels is fully reversible. Thus, only data of the heating cycles are shown. The swelling ratio $Q$ is calculated from the hydrodynamic radii as $Q=R_{\mathrm{h}}\left(10^{\circ} \mathrm{C}\right) / R_{\mathrm{h}}\left(40^{\circ} \mathrm{C}\right)$ and the VPTTs are determined from the inflection points of the swelling curves.

\section{Static light scattering}

Static light scattering (SLS) was performed to determine the form factors of microgels. Highly diluted samples were probed with a laser of the wavelength $\lambda=404 \mathrm{~nm}$ mounted on a closed goniometer from SLS-Systemtechnik $\mathrm{GmbH}$ and connected to a controllable thermostat to adjust the temperature of the toluene bath that surrounds the sample. The scattering intensities were measured from $15^{\circ}$ to $150^{\circ}$ and corrected for the background. The resulting form factors were fitted with a model introduced by Berndt et al. describing a core-shell morphology with fuzzy interfaces [14].

\section{Results and discussion}

An effective way to lower the VPTT of microgels is by increasing the hydrophobicity of the co-polymeric network, which can even lead to microgels insoluble in water. NtBAM is well-known to lower the LCST of polymers, and thus, the VPTT of microgels when being co-polymerized with NIPAM [37-41]. In the Supplementary Information, we show that the microgels possess distinct VPTTs depending on the amount of NtBAM: the more NtBAM in the network, the lower is the VPTT. Additionally, we demonstrate that in contrast to conventional p(NIPAM-coNtBAM) microgels, the generation of hollow p(NIPAM-coNtBAM) microgels is precluded as the microgels lose their integrity and aggregation occurs during the core removal and the purification procedure.

For these reasons, we introduce an alternative LCSTlowering co-monomer, namely DAAM. In contrast to NtBAM, DAAM is well soluble in water, which improves the reproducibility of the syntheses. Only few studies on the co-polymerization of DAAM with acrylamides are known [33-36]. To the best of our knowledge, this is the first study of conventional and hollow microgels based on p(NIPAM-co-DAAM) aiming at a decreased VPTT.

The precipitation polymerization of NIPAM initiated by a persulfate initiator in absence of a cross-linking agent results in the so-called ultra-low cross-linked (ULC) microgels $[50,51]$. P(NIPAM-co-DAAM) ULC microgels were synthesized and investigated by means of ${ }^{1} \mathrm{H}$ NMR spectroscopy to conclude on a successful DAAM incorporation 
in the polymeric networks (see Supplementary Information, Fig. S.6).

On the basis of a successful DAAM co-polymerization, syntheses of conventionally cross-linked microgels with various NIPAM:DAAM ratios were performed. This aims to determine an appropriate monomer composition to lower the VPTT of the microgels below room temperature without completely suppressing the thermoresponsive character in water. Two different molar NIPAM:DAAM:BIS ratios were chosen: 0.65:0.30:0.05 (referred to as 30\%DAAM) and 0.30:0.65:0.05 (65\%DAAM), meaning that both microgel types comprise a cross-linking density of $5 \mathrm{~mol} \%$.

Figure 2 illustrates the hydrodynamic radii, $R_{\mathrm{h}}$, as a function of temperature, $T$, determined from angle-dependent dynamic light scattering. As the first observation can be stated that the radius of the 65\%DAAM microgels (diamonds) is constant throughout the investigated temperature range. The thermoresponsive behavior gets suppressed as the amount of DAAM co-monomer is rather high. In contrast, the 30\%DAAM microgels (circles) reveal a typical response to temperature changes: upon cooling the microgels swell by increasing their size. A swelling ratio of $Q(30 \% \mathrm{DAAM})=1.87 \pm 0.04$ (with $Q=$ $\left.R_{\mathrm{h}}\left(10^{\circ} \mathrm{C}\right) / R_{\mathrm{h}}\left(40^{\circ} \mathrm{C}\right)\right)$ is a typical value for $5 \mathrm{~mol} \%$ crosslinked pNIPAM microgels [42]. The microgels possess a VPTT of about $15^{\circ} \mathrm{C}$, which fulfills the requirements of being below room temperature and maintaining the thermoresponsivity in water.

However, the p(NIPAM-co-DAAM) microgels possess rather limited stability. Figure 4a shows that the 65\%DAAM sample is stable at room temperature. It can be considered as stable latex suspension. In contrast, the thermosensitive 30\%DAAM microgels are not stable above the VPTT (Fig. 4b). Longer storage at room temperature leads to irreversible aggregation.

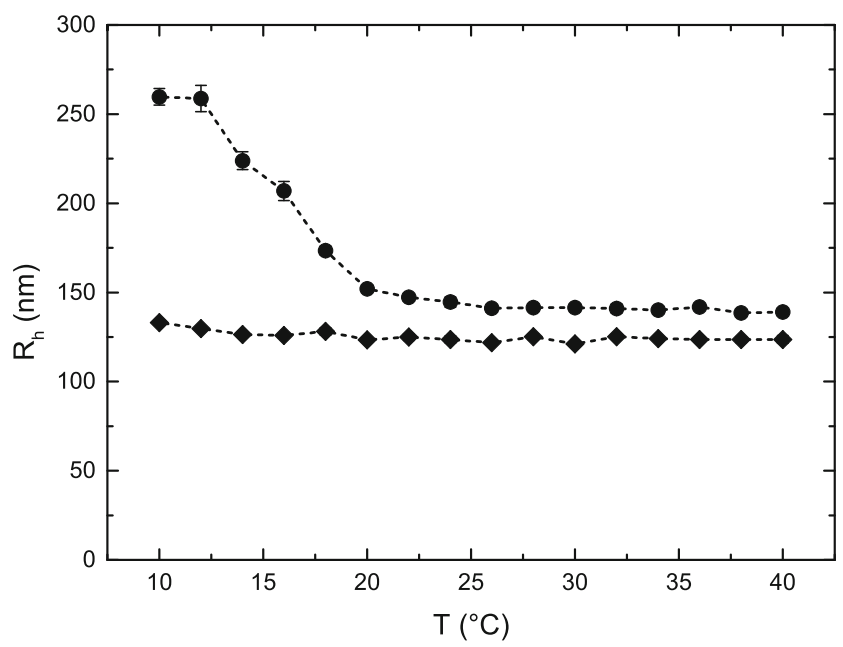

Fig. 2 Hydrodynamic radius, $R_{\mathrm{h}}$, as a function of the temperature, $T$, of 30\%DAAM (circles) and 65\%DAAM (diamonds) microgels
For all microgels presented below, the monomer composition of the 30\%DAAM microgels is chosen. In the following, two methods are tested to improve the stability of the microgels in the collapsed state: (i) via the addition of a surfactant during the precipitation polymerization to electrostatically stabilize the polymeric globules at an earlier time point (referred to as 30\%DAAM-Electrostatic) [10] and (ii) by adding additional steric interactions via a swollen pNIPAM shell as a periphery to the microgels (30\%DAAMSteric) [19].

Figure 3 presents the temperature-dependent sizes of the microgels resulting from both methods in comparison to the $30 \%$ DAAM microgels (circles) presented above. The microgels synthesized in presence of a surfactant, SDS, (30\%DAAM-Electrostatic; squares) are considerably smaller. This results from an enhanced colloidal stabilization during the nucleation stage of the polymerization [10]. The VPTT as well as the swelling ratio of $Q(30 \%$ DAAMElectrostatic $)=1.90 \pm 0.04$ are not affected by the presence of SDS.

However, upon purification, the SDS is removed from the microgels, which results in a loss of electrostatic stabilization and thus, in irreversible aggregation of the microgels (see Fig. 4c).

The core-shell microgels with an additional pNIPAM shell as outer periphery (30\%DAAM-Steric; triangles) are a bit larger throughout the whole temperature range when compared to the 30\%DAAM microgels. This indicates a successful addition of the pNIPAM network. A marginal swelling of $5 \mathrm{~nm}$ can be observed below the VPTT of pure pNIPAM $\left(32^{\circ} \mathrm{C}\right)$. The main VPTT is still observed at about $15^{\circ} \mathrm{C}$ and the swelling ratio is with $Q(30 \%$ DAAMSteric $)=1.89 \pm 0.04$ again comparable to pNIPAM-based microgels with similar cross-linking densities.

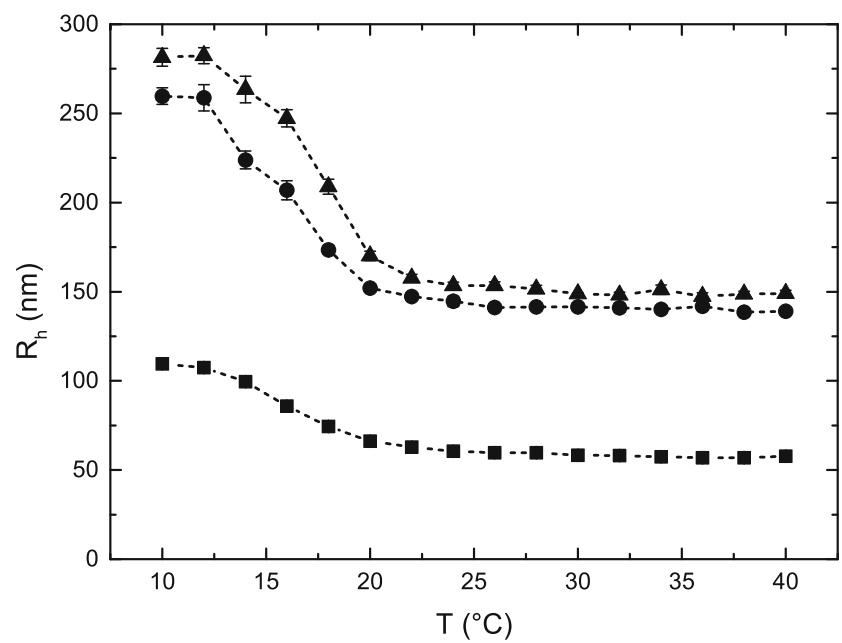

Fig. 3 Hydrodynamic radius, $R_{\mathrm{h}}$, as a function of the temperature, $T$, of the 30\%DAAM (circles), 30\%DAAM-Electrostatic (squares), and $30 \%$ DAAM-Steric (triangles) 
Finally, a successful steric stability can be concluded. A solution of the 30\%DAAM-Steric microgels can be stored at room temperature without the appearance of aggregates (see Fig. 4d).

The improved stability of the p(NIPAM-co-DAAM)-core pNIPAM-shell microgels marks the starting point for the synthesis of hollow microgels with a VPTT below room temperature. In a first step, microgels are synthesized with a silica nanoparticle as a core surrounded by an inner $\mathrm{p}$ (NIPAM-co-DAAM) shell, which is again surrounded by an outmost pNIPAM shell as depicted in Fig. 1 . The corresponding hollow double-shell microgels with a solvent-filled cavity are obtained by core dissolution. The cavity is then surrounded by two distinct networks: an outer pNIPAM network for steric stability and an inner $\mathrm{p}$ (NIPAM-co-DAAM) network. The latter is deswollen at room temperature and permits to control the permeability toward the cavity for future encapsulation experiments as predicted by computer simulations [3].

Figure $4 \mathrm{e}$ and $\mathrm{f}$ show that solutions of the core-doubleshell and corresponding hollow microgels, respectively, are stable at room temperature. This is a great advantage when compared to the hollow p(NIPAM-co-NtBAM) microgels, which have been presented in the Supplementary Information.

Figure 5 shows the hydrodynamic radius, $R_{\mathrm{h}}$, as a function of temperature, $T$, of the core-double-shell (full circles) and corresponding hollow microgels (open circles). The sacrificial silica cores possess a radius of $60 \mathrm{~nm}$. The core-double-shell microgels have the aimed characteristics: they exhibit temperature-sensitivity with a VPTT below room temperature and a swelling ratio of $Q$ (Core-doubleshell $)=2.06 \pm 0.05$. Considering the masses employed during the synthesis yields in a theoretical collapsed shell thickness of the outer pNIPAM network of $3 \mathrm{~nm}$.

After core removal, the hollow microgels possess a highly comparable swelling behavior. The sizes of both

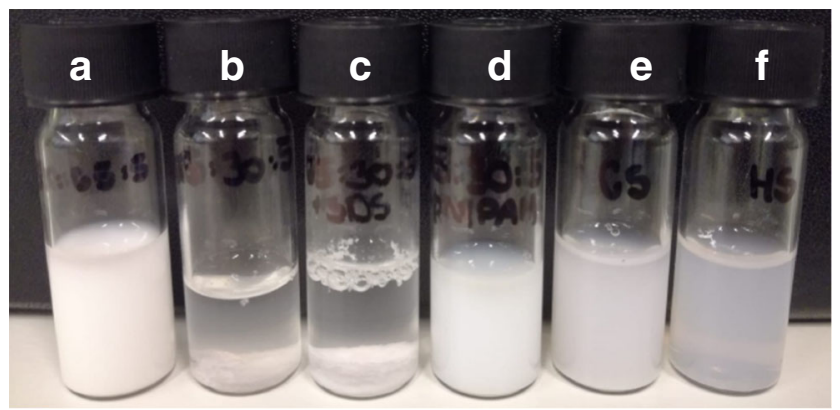

Fig. 4 Solutions of a 65\%DAAM, b 30\%DAAM, c 30\%DAAMElectrostatic, d 30\%DAAM-Steric, e silica-core p(NIPAM-coDAAM)-shell pNIPAM-shell microgels, and $\mathbf{f}$ corresponding hollow microgels stored at room temperature. b and $\mathbf{c}$ reveal irreversible aggregation, while all other microgel solutions are colloidally stable core-double-shell and hollow microgels correspond in the deswollen state. However, the nanocapsules are smaller in the swollen state. This is most likely related to the cavity. The latter gives the polymeric network an additional possibility to swell: not only toward the outer, but also toward the inner periphery $[19,20,43]$. The swelling ratio of $Q$ (Hollow $)=2.00 \pm 0.06$ agrees with previous studies on hollow pure pNIPAM microgels with a cross-linking density of $5 \mathrm{~mol} \%$ [42]. The VPTT is slightly shifted toward higher temperature when compared to the core-double-shell microgels. This has also been observed previously for pure pNIPAM microgels and may arise from an alteration of the solvent-polymer interactions resulting from a network loosening due to the core removal [20].

Structural analysis of the microgels in bulk is required to verify the hypothesis of a partial internal swelling and ensure that the cavity is still intact. Due to the sizerange of these microgels, static light scattering was chosen to determine the form factors of the hollow p(NIPAMco-DAAM) microgels in their swollen $\left(\right.$ at $10^{\circ} \mathrm{C}$ ) and deswollen (at $30^{\circ} \mathrm{C}$ ) states to investigate the internal structures.

Figure 6a shows the scattering intensities as a function of the scattering vector, $q$, of the hollow microgels at $30^{\circ} \mathrm{C}$ (diamonds) and $10^{\circ} \mathrm{C}$ (circles). The fact that the curves approach a flat plateau for low- $q$ indicates that the microgels are colloidally stable and aggregation is prevented. The form factor model developed by Berndt et al. [14] (solid black lines) was chosen as best fits to reproduce the experimental data. It describes a core-shell morphology with fuzzy interfaces.

In the Supplementary Information (Fig. S.7), we show that the simpler model of a fuzzy sphere by Stieger et al. [52] cannot fit the scattering intensities. The scattering

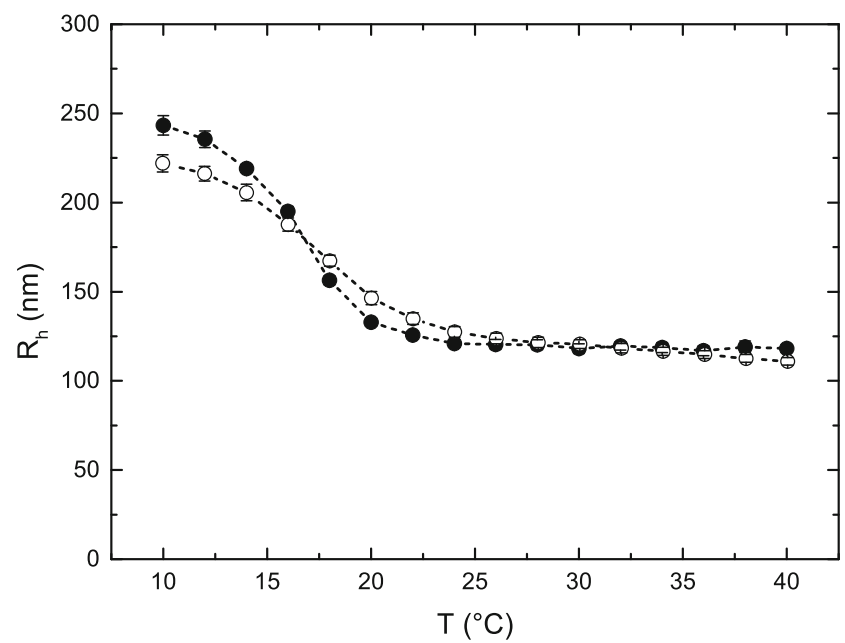

Fig. 5 Hydrodynamic radius, $R_{\mathrm{h}}$, as a function of the temperature, $T$, of silica-core p(NIPAM-co-DAAM)-shell pNIPAM-shell (full circles) and corresponding hollow microgels (open circles) 

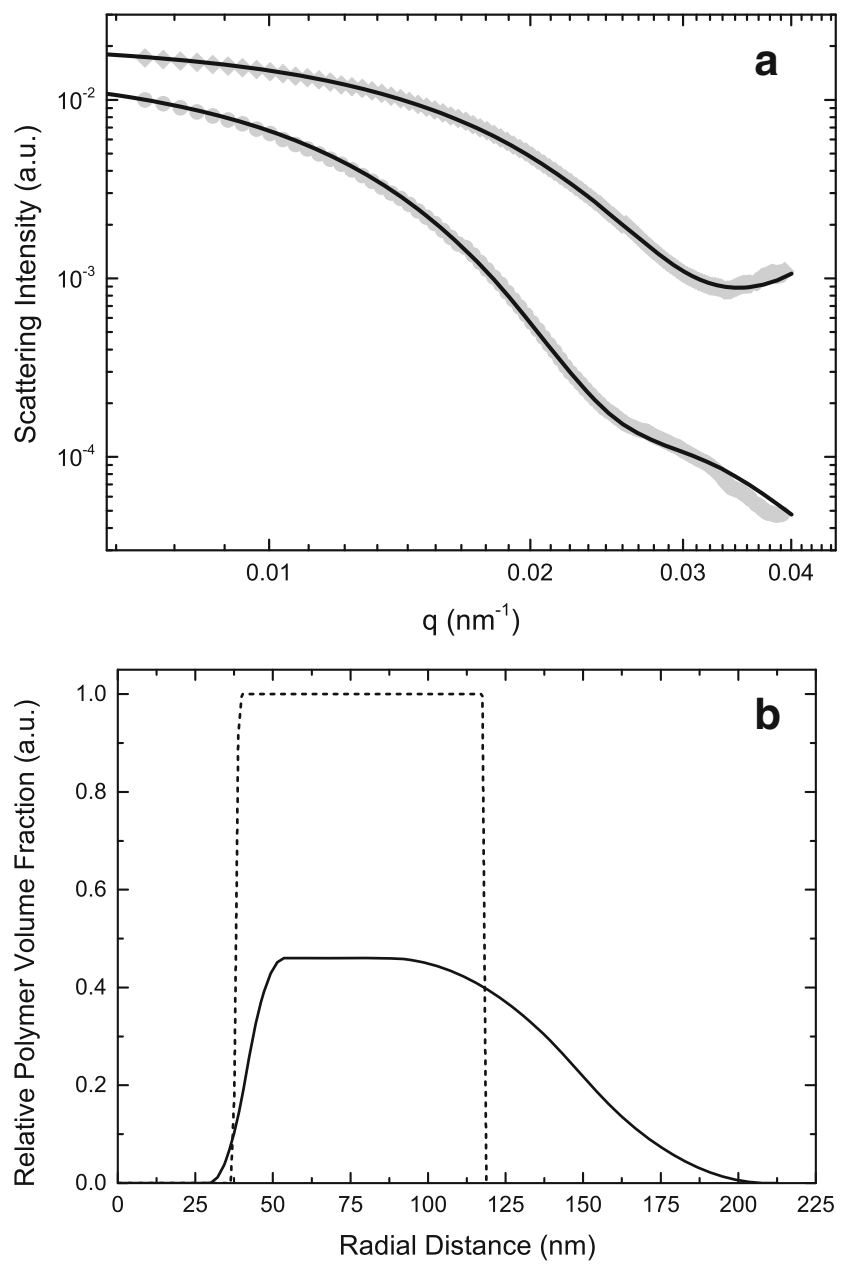

Fig. 6 a Scattering intensity as a function of the scattering vector, $q$, of the hollow $\mathrm{p}$ (NIPAM-co-DAAM) microgels probed at $30^{\circ} \mathrm{C}$ (diamonds) and $10^{\circ} \mathrm{C}$ (circles). The fits from the fuzzy core-shell model are represented by the black lines [14]. b Radial density distributions obtained from the fits at $10^{\circ} \mathrm{C}$ (solid lines) and $30^{\circ} \mathrm{C}$ (dashed lines)

data are only reproduced appropriately when using a model describing a hollow sphere.

Figure $6 \mathrm{~b}$ illustrates the radial density profiles obtained from the fits with the fuzzy core-shell model shown in Fig. 6a. Above the VPTT, at $30^{\circ} \mathrm{C}$, (dashed line) a box-like profile with an internal cavity is obtained. It corresponds to a collapsed polymer shell with constant density and a cavity with a radius of $38 \mathrm{~nm}$. The total size is $119 \mathrm{~nm}$ with a polydispersity of $15 \%$. The swollen pNIPAM shell cannot be identified. This may result from an interpenetration into the deswollen p(NIPAM-co-DAAM) network in combination with the small thickness [53-55].

Below the VPTT, at $10^{\circ} \mathrm{C}$, (solid line) the structure of the microgels is as well hollow. The microgels are swollen as the relative polymer volume fraction is considerably lower when compared to the collapsed state. The swollen microgels possess decaying polymer densities toward the inner and outer periphery of the shell. This means, as hypothesized, that the shell expands toward the external periphery and toward the cavity, whose size is slightly decreased. The microgels possess a total radius of $210 \mathrm{~nm}$ with a polydispersity of $20 \%$ and a cavity size of $30 \mathrm{~nm}$.

From our form factor analysis, it can be concluded that the microgels are colloidally stable in water at all swelling states. Additionally, transmission electron micrographs shown in Fig. S.8 of the Supplementary Information visualize both core-shell and hollow microgels. Finally, hollow $\mathrm{p}$ (NIPAM-co-DAAM) microgels with a VPTT below room temperature were successfully synthesized. The cavity is maintained at all swelling states, which is of great importance for future encapsulation experiments.

\section{Conclusions}

In summary, the synthesis of hollow pNIPAM-based microgels with a VPTT below room temperature is rather challenging with respect to the colloidal stability of the microgels when being deswollen in water. Hollow p(NIPAM-coNtBAM) microgels revealed irreversible aggregation when stored at room temperature. Due to poor reproducibility of the syntheses, the VPTT-lowering co-monomer was exchanged to DAAM.

We report on the first synthesis of p(NIPAM-co-DAAM) microgels with low VPTT. The attempts to resolve the stability issues by increasing the electrostatic or steric interactions revealed that the addition of an outer pNIPAM periphery to the $\mathrm{p}$ (NIPAM-co-DAAM) microgels is appropriate to prevent aggregation of the polymeric networks.

On the basis of these findings, stable silica-core $\mathrm{p}(\mathrm{NI}-$ PAM-co-DAAM)-shell microgels have been synthesized with an outmost pNIPAM shell, which is swollen at room temperature. This ensures sufficient steric interactions between the microgels even when the p(NIPAM-coDAAM) network is collapsed. The corresponding hollow nanocapsules were characterized by means of dynamic and static light scattering. Form factor analysis demonstrated that the cavity of the hollow microgels persists at all swelling states, which makes them suitable candidates for future encapsulation experiments. The present study in combination with previous reports [18-20] on hollow microgels enables the possibility to synthesize stable hollow microgels and tune the VPTT as desired from below room temperature to well above body temperature.

Acknowledgements The authors acknowledge financial support from the SFB 985 "Functional Microgels and Microgel Systems" of Deutsche Forschungsgemeinschaft within project A3.

Funding Information Open Access funding provided by Projekt DEAL. 


\section{Compliance with ethical standards}

Conflict of interests The authors declare that they have no conflict of interest.

Availability of data and material Additional supporting research data for this article may be accessed at no charge at https://hdl.handle.net/ 21.11102/593ab6d4-75b4-11ea-937a-e41f1366df48.

Open Access This article is licensed under a Creative Commons Attribution 4.0 International License, which permits use, sharing, adaptation, distribution and reproduction in any medium or format, as long as you give appropriate credit to the original author(s) and the source, provide a link to the Creative Commons licence, and indicate if changes were made. The images or other third party material in this article are included in the article's Creative Commons licence, unless indicated otherwise in a credit line to the material. If material is not included in the article's Creative Commons licence and your intended use is not permitted by statutory regulation or exceeds the permitted use, you will need to obtain permission directly from the copyright holder. To view a copy of this licence, visit http:// creativecommonshorg/licenses/by/4.0/.

\section{References}

1. Thorne JB, Vine GJ, Snowden MJ (2011) Colloid Polym Sci 289:625

2. Dirksen M, Dargel C, Meier L, Brändel T, Hellweg T (2020) Colloid Polym Sci 298:505. https://doi.org/10.1007/s00396-02004632-5

3. Richtering W, Potemkin II, Rudov AA, Sellge G (2016) Nanomedicine 11:2879

4. Oh JK, Drumright R, Siegwart DJ, Matyjaszewski K (2008) Prog Polym Sci 33:448

5. Oh JK, Siegwart DJ, Lee HI, Sherwood G, Peteanu L, Hollinger JO, Kataoka K, Matyjaszewski K (2007) J Am Chem Soc 129:5939

6. Chen J, Wu M, Veroniaina H, Mukhopadhyay S, Li J, Wu Z, Wu Z, Qi X (2019) Polym Chem 10:4031

7. Pich A, Richtering W (2010) Adv Polym Sci 234:1

8. Karg M, Pich A, Hellweg T, Hoare T, Lyon LA, Crassous JJ, Suzuki D, Gumerov RA, Schneider S, Potemkin II, Richtering W (2019) Langmuir 35:6231

9. Pelton R (2000) Adv Colloid Interf Sci 85:1

10. McPhee W, Tam KC, Pelton R (1993) J Colloid Interface Sci $156: 24$

11. Debord SB, Lyon LA (2003) J Phys Chem B 107:2927

12. Garcia A, Marquez M, Cai T, Rosario R, Hu Z, Gust D, Hayes M, Vail SA, Park CD (2007) Langmuir 23:224

13. Islam MR, Ahiabu A, Li X, Serpe MJ (2014) Sensors 14:8984

14. Berndt I, Pedersen JS, Richtering W (2006) Angewandte Chemie - International Edition 45:1737

15. Karg M, Pastoriza-Santos I, Liz-Marzán LM, Hellweg T (2006) ChemPhysChem 7:2298

16. Cors M, Wrede O, Genix AC, Anselmetti D, Oberdisse J, Hellweg $\mathrm{T}$ (2017) Langmuir 33:6804

17. Oberdisse J, Hellweg T (2020) Colloid Polym Sci, https://doi.org/10.1007/s00396-020-04629-0

18. Dubbert J, Nothdurft K, Karg M, Richtering W (2015) Macromol Rapid Commun 36:159

19. Schmid AJ, Dubbert J, Rudov AA, Pedersen JS, Lindner P, Karg M, Potemkin II, Richtering W (2016) Sci Rep 6:22736
20. Brugnoni M, Scotti A, Rudov AA, Gelissen APH, Caumanns T, Radulescu A, Eckert T, Pich A, Potemkin II, Richtering W (2018) Macromolecules 51:2662

21. Nayak S, Gan D, Serpe MJ, Lyon LA (2005) Small 1:416

22. Dubbert J, Honold T, Pedersen JS, Radulescu A, Drechsler M, Karg M, Richtering W (2014) Macromolecules 47:8700

23. Sukhorukov G, Fery A, Möhwald H (2005) Prog Polym Sci 30:885

24. Lou XW, Archer LA, Yang Z (2008) Adv Mater 20:3987

25. Landfester K (2009) Angewandte Chemie - International Edition 48:4488

26. Cheng CJ, Chu LY, Ren PW, Zhang J, Hu L (2007) J Colloid Interface Sci 313:383

27. Sanson N, Rieger J (2010) Polym Chem 1:965

28. Choi CH, Jung JH, Kim DW, Chung YM, Lee CS (2008) Lab Chip 8:1544

29. Masoud H, Alexeev A (2012) ACS Nano 6:212

30. Werner JG, Deveney BT, Nawar S, Weitz DA (2018) Adv Funct Mater 28:1303385

31. Su Y, Ojo OF, Tsengam IKM, He J, McPherson GL, John VT, Valla JA (2018) Langmuir 34:14608

32. Moncho-Jordá A, Germán-Bellod A, Angioletti-Uberti S, Adroher-Benítez I, Dzubiella J (2019) ACS Nano 13:1603

33. Taylor LD, Cerankowski LD (1975) J Polym Sci Polym Chem Ed 13:2551

34. Ni CH, Zhu XX, Wang QL, Zeng XY (2007) Chin Chem Lett 18:79

35. Xing XM, Liu GM, Ding YW, Zhang GZ (2014) Chin J Polym Sci 32:531

36. Guo Y, Song R, Feng R, Dai G, Liang Y, Pu D, Zhang X, Ye Z (2019) J Appl Polym Sci 136:47051

37. Hertle Y, Zeiser M, Hasenöhrl C, Busch P, Hellweg T (2010) Colloid Polym Sci 288:1047

38. Yi YD, Bae YC (1998) J Appl Polym Sci 67:2087

39. Liu HY, Zhu XX (1999) Polymer 40:6985

40. Debord JD, Lyon LA (2003) Langmuir 19:7662

41. Selezneva II, Gorelov AV, Rochev YA (2006) Bull Exp Biol Med 2:538

42. Scotti A, Denton AR, Brugnoni M, Houston JE, Schweins R, Potemkin II, Richtering W (2019) Macromolecules 52:3995

43. Nickel AC, Scotti A, Houston JE, Ito T, Crassous J, Pedersen JS, Richtering W (2019) Nano Lett 19:8161

44. Geisel K, Rudov AA, Potemkin II, Richtering W (2015) Langmuir 31:13145

45. Schulte MF, Scotti A, Gelissen APH, Richtering W, Mourran A (2018) Langmuir 34:4150

46. Song X, Bao B, Tao J, Zhao S, Han X, Liu H (2019) J Phys Chem C 123:1828

47. Scotti A, Brugnoni M, Rudov AA, Houston JE, Potemkin II, Richtering W (2018) J Chem Phys 148:174903

48. Stöber W, Fink A, Bohn E (1968) J Colloid Interface Sci 26:62

49. Burchard W, Richtering W (1989) Prog Collied Polym Sci 80:151

50. Gao J, Frisken BJ (2003) Langmuir 19:5212

51. Brugnoni M, Nickel AC, Kröger LC, Scotti A, Pich A, Leonhard K, Richtering W (2019) Polym Chem 10:2397

52. Stieger M, Richtering W, Pedersen JS, Lindner P (2004) J Chem Phys 120:6197

53. Gan D, Lyon LA (2001) J Am Chem Soc 123:7511

54. Jones CD, McGrath JG, Lyon LA (2004) J Phys Chem B 108:12652

55. Cors M, Wiehemeier L, Wrede O, Feoktystov A, Cousin F, Hellweg T, Oberdisse J (2020) Soft Matter 16:1922

Publisher's note Springer Nature remains neutral with regard to jurisdictional claims in published maps and institutional affiliations. 